\title{
Analysis of Carbonyl Value of Frying Oil by Fourier Transform Infrared Spectroscopy
}

\author{
Han Zhang ${ }^{1}$, Jinkui $\mathrm{Ma}^{2}$, Yelian Miao ${ }^{3}$, Tomohiro Tuchiya ${ }^{1}$ and Jie Yu Chen ${ }^{1 *}$ \\ ${ }^{1}$ Akita Prefectural University, Akita, 010-0195 Japan \\ ${ }^{2}$ Zhaoqing University, Zhaoqing, Guangdong, 526061, China \\ ${ }^{3}$ Nanjing University of Technology, Nanjing, 211816 China
}

\begin{abstract}
A rapid method for determining the carbonyl value of frying oils has been developed using Fourier-transform infrared (FTIR) spectroscopy and chemometrics. One hundred and fifty-six frying oils with different carbonyl values were collected from an actual potato frying process. FTIR spectra in the range of 4000-650 $\mathrm{cm}^{-1}$ were scanned with a FTIR spectroscopy apparatus using the attenuated total reflectance (ATR) method. A good calibration model was obtained using the partial least-squares (PLS) regression method with full cross validation for predicting the carbonyl value of frying oils. For the model, the coefficients of determination $\left(R^{2}\right)$, standard errors of cross validation (SECV) and standard errors of prediction (SEP) were $0.99,1.87 \mu \mathrm{mol} \mathrm{g}^{-1}$ and $1.93 \mu \mathrm{mol} \mathrm{g}^{-1}$, respectively. Moreover, standard deviation ratios of reference data in the validation sample set to the SEP were higher than 3 . This study shows that the carbonyl value of frying oils can be successfully determined to a high accuracy using FTIR spectroscopy combined with PLS regression.
\end{abstract}

Key words: frying oil, carbonyl value, degradation, Fourier-transform infrared spectroscopy, partial least squares regression

\section{INTRODUCTION}

The quality of fried food is closely related to the quality of the frying oil ${ }^{1)}$. During frying, oil is subjected to prolonged periods of heating at high temperatures in the presence of air and water, which leads to a wide range of complex chemical reactions, such as thermal oxidation, hydrolysis, and polymerization ${ }^{2,3)}$. The compounds generated from these chemical reactions not only have negative effects on flavor but also form undesirable constituents in fried foods ${ }^{4)}$. Therefore, controlling the quality of frying oil is very important and evaluating that quality rapidly is imperative.

Analysis of the carbonyl value(CV) is useful for evaluating the degradation of frying oils, because it indicates the quantity of carbonyl compounds. These possess the most rancid and unpleasant flavors of the secondary products of oil oxidation formed by the degradation of hydro-peroxides during the frying process ${ }^{5-8)}$.

The CV analysis methods used most widely are based on the colorimetric measurement of 2,4-dinitrophenylhydrazones in an alkali solution ${ }^{9,10)}$, 2-propanol (2-PrOH)instead of benzene ${ }^{8)}$ and commercial 1-butanol $(1-\mathrm{BuOH})^{11)}$. These methods can be used to quantify the CV of frying oils accurately, but are expensive and time-consuming.
Fourier-transform infrared (FTIR) spectroscopy has proved to be a good alternative to standard wet analytical techniques for determining key quality parameters in many fields ${ }^{12-14)}$. It has the advantages of rapid acquisition of spectral data, is easy to operate and needs no complex sample preparation ${ }^{15}$. Previous FTIR studies have reported identifying a variety of food adulteration problems using experimental and statistical methods ${ }^{16-18)}$. More recently, FTIR spectroscopy has been used to determine how edible vegetable oils, adulterated with used frying oil, deteriorat$\mathrm{ed}^{19,20)}$. These studies showed the ability of FTIR spectroscopy to successfully monitor the FFA (Free fatty acid content) of heated edible and frying oils, but no work has been reported on analyzing the $\mathrm{CV}$ of frying oils.

The objective of the present study was therefore to investigate whether FTIR spectroscopy combined with chemometrics could be used for prediction of the CV of a large number of deep frying oils samples collected from two types of rapeseed oils.

\footnotetext{
*Correspondence to: Jie Yu Chen, Faculty of Bioresource Sciences, Akita Prefectural University, 241-438 Kaidobata-Nishi Nakano Shimoshinjo Akita City, 010-0195, JAPAN

E-mail: jiey_chen@akita-pu.ac.jp

Accepted December 16, 2014 (received for review September 9, 2014)

Journal of Oleo Science ISSN 1345-8957 print / ISSN 1347-3352 online

http://www.jstage.jst.go.jp/browse/jos/ http://mc.manusriptcentral.com/jjocs
} 


\section{EXPERIMENTAL PROCEDURES}

\subsection{Sample preparation}

Two types of rapeseed oil (commercially refined canola oil from the Nisshin Oillio Group Ltd, Tokyo, Japan and unrefined rapeseed Kizakinonatane oil from Akita New Bio Farm Co. Ltd, Akita, Japan) were used as the frying oil samples. Frozen par-fried French fries in an institutional pack were purchased from a local supermarket and used for deep-frying. The frying was conducted in a restaurantstyle stainless steel electric fryer TF-40A (Taiji \& Co. Ltd., Kanagawa, Japan) at frying temperatures of 180, 200 or $220^{\circ} \mathrm{C}$. Batches of $100 \mathrm{~g}$ of frozen French fries were fried for $3 \mathrm{~min}$ at 22-min intervals, when the temperature of the oil had reached the desired temperature. This continued for a period of $7 \mathrm{~h}$ each day for 4 consecutive days. This was equivalent to frying 17 batches per day and therefore 68 batches for the whole experiment. During the frying process, $200 \mathrm{ml}$ of heated oil was drawn off every $3.5 \mathrm{~h}$ and stored at $-18^{\circ} \mathrm{C}$ until analysis of carbonyl values and acquisition of IR spectral data. The frying experiments were carried out once using canola oil and twice using rapeseed Kizakinonatane oil under the two method: without replenishment (no fresh oil addition) and with frequent replenishment of oil (200 ml fresh oil addition after having gathered the frying oil sample of $200 \mathrm{ml}$ ). A total of 156 frying oil samples, degraded to different degrees, were obtained from the food frying process.

\subsection{Reference analysis}

The CVs of the frying oil samples were determined according to the Japan Oil Chemists' Society (JOCS) Official Method Tentative 13-2003 Carbonyl Value (Butanol Method) developed by Endo et al. ${ }^{11,21)}$. All CV analysis results are expressed in $\mu \mathrm{mol} \mathrm{g}^{-1}$.

\subsection{FTIR spectral acquisition}

Spectroscopic data from the frying oil samples were acquired using an infrared spectrometer(Nicolet 6700 FT-IR, Thermo Fisher Scientific K.K, Waltham, MA, USA) equipped with an attenuated total reflection (ATR) smart sampling accessory with a temperature controller. A small amount of the oil sample was uniformly deposited on the crystal surface of the ATR accessory (Specac Inc., Woodstock, GA, USA), equipped with a ZnSe 11 reflection crystal. Analyses were carried out at room temperature. Spectra were acquired (100 scans/sample or background) in the wavenumber range of $4000-650 \mathrm{~cm}^{-1}$ at a spectral resolution of $4 \mathrm{~cm}^{-1}$, and the data exported by OPUS Ver. 6.0 (Bruker Optics, Billerica, MA, USA) software in ASCII compatible format. For each sample, the absorbance spectrum was collected against a background obtained with a dry and empty ATR cell. Three spectra per sample were recorded. After acquiring each spectrum, the ATR crystal was cleaned with a cellulose tissue soaked in hexane and then rinsed with acetone. All spectral data exported by the OPUS software in ASCII compatible format were then imported to Unscrambler analytical software ver. 7.6 (CAMO, Oslo, Norway).

\subsection{Statistical Analysis}

The calibration models were created by partial least square (PLS) regression from the raw FTIR spectra and its first and second derivatives, and the optimum number of PLS factors used for prediction was determined by full cross validation. The number of significant PLS factors was chosen by using a predicted residual error sum of squares (SECV) value for every possible factor. The SECV value was the sum of the squared differences between the predicted and the known carbonyl values. This was calculated by building calibration models with different numbers of factors and then predicting some samples of known carbonyl concentration against the model. The quality of the calibration models can be described by the squared correlation coefficient $\left(\mathrm{R}^{2}\right)$ and the standard error of calibration (SEC), and was validated using the validation sample set that was not used for the calibration development. The correlation coefficient of prediction $(r)$ and root-mean square of the prediction (SEP) were used to choose the best model. SEP measures how well the model can predict samples in a validation set. The best calibration model to be used for prediction was the one with the highest value of $r$ and lowest value for SEP.

The first and second derivative spectra ${ }^{22,23)}$ were obtained using the Savitsky-Golay method ${ }^{24)}$ with a segment of 8 points and gap of 0 points. The PLS regression and derivative mathematical treatments were performed using the Unscrambler software.

The 156 oil samples obtained from the frying process were divided into calibration and validation sets as follows. Initially, samples within the parent set were sorted according to the CV determined chemically. Starting with the lowest CV sample, the first and third samples were assigned to the calibration set, and the second sample to the validation set. The next group of three samples was similarly assigned, and so on, until the last group. Thus, 104 samples were included in the calibration set and 52 in the validation set. Statistics for the CV of the frying oil samples selected for the calibration and validation sets are shown in Table 1.

\section{RESULTS AND DISCUSSION}

\subsection{Spectral features of FTIR}

Figure 1 shows the FTIR spectra of two fresh oils (canola oil and rapeseed Kizakinonatane oil). To the naked eye, the entire range of spectra looks very similar for the two oils. These spectra showed absorption bands typically charac- 
Table 1 Characteristics of reference data for calibration and validation sets.

\begin{tabular}{ccccccccc}
\hline & \multicolumn{3}{c}{ Calibration set $(\mathrm{n}=104)$} & & \multicolumn{3}{c}{ Validation set $(\mathrm{n}=52)$} \\
\cline { 2 - 4 } \cline { 7 - 8 } & Mean & Range & SD & & Mean & Range & SD \\
\hline $\mathrm{CV}\left(\mu \mathrm{mol} \mathrm{g}^{-1}\right)$ & 16.28 & $1.99-43.90$ & 11.21 & & 16.17 & $1.99-41.47$ & 11.04 \\
\hline
\end{tabular}

SD: standard deviation

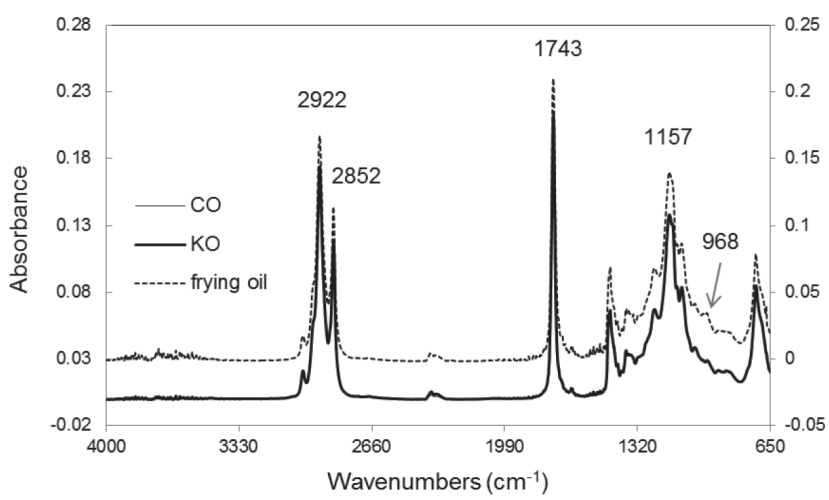

Fig. 1 FTIR spectra of two fresh oils (Canola oil and rapeseed Kizakinonatane oil) and one used Canola frying oil samples.

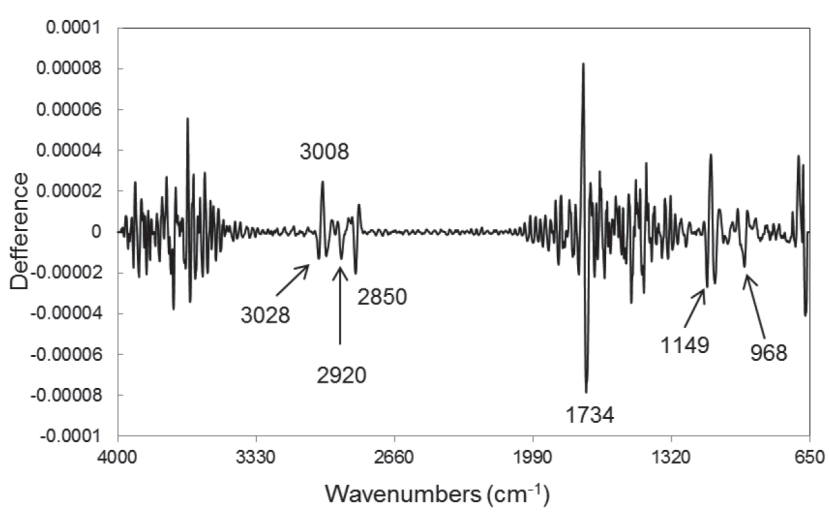

Fig. 2 The difference spectrum between two FTIR second derivative spectra of the high-CV oil sample (deteriorated Canola oil) and the fresh Canola oil sample.

teristic of those reported in previous studies ${ }^{18,25-27)}$. The most prominent absorption band at $1743 \mathrm{~cm}^{-1}$ can be assigned to the $\mathrm{C}=\mathrm{O}$ stretching of aliphatic esters ${ }^{18,25-27)}$.
The strong bands at around 2922 and $2852 \mathrm{~cm}^{-1}$ can be ascribed to the asymmetrical and symmetrical C-H stretching vibrations of $\mathrm{CH}_{2}$ groups. The band at around $1157 \mathrm{~cm}^{-1}$ may be assigned to the stretching of the $\mathrm{C}-\mathrm{O}$ bonds of aliphatic esters or $\mathrm{CH}_{2}$ bending vibrations ${ }^{18,25-27)}$. The FTIR spectrum (dotted line in Fig. 1) from used Canola frying oil resembled that of the fresh oils. However, if one examines the difference spectrum between two FTIR second derivative spectra of the high-CV oil (deteriorated Canola oil) sample and the fresh Canola oil sample (Fig. 2) closely, differences between deteriorated and fresh Canola oil samples are observed at these typical absorption bands around 3028, 3008, 2920, 2850, 1734 and $1149 \mathrm{~cm}^{-1}$. In addition, the absorption at $968 \mathrm{~cm}^{-1}$ observed in the FTIR second derivative spectrum of the used frying oil, which may be due to the $\mathrm{C}-\mathrm{H}$ out-of-plane deformation of isolated trans double bonds or some trans conjugated unsaturated fatty acids ${ }^{18,25-27)}$. The peak intensity at $968 \mathrm{~cm}^{-1}$ exhibited a slight increase in used frying oils compared with the fresh oil. From these observations, it can be seen that much information about the degradation of frying oil can be obtained from FTIR spectra.

\subsection{Calibration models for CV}

As shown in Table 2, a total of 3 PLS calibration models were developed for analyzing the $\mathrm{CV}$ of frying oils using the calibration and validation frying oil sample sets based on raw, first and second derivative spectra. There were very strong correlations between actual values and IR-predicted values in the calibration models, with $\mathrm{R}^{2}$ values greater than 0.97. When the SEC, SECV and SEP values of the calibration models were compared, as shown in Table 2, the results based on the first or second derivative spectra were better than those based on the raw spectra. Because there were differences in the viscosity of frying oil samples, the baseline offset and slopes in the spectra may have affected

Table 2 PLS analysis results for CVs of frying oil samples.

\begin{tabular}{|c|c|c|c|c|c|c|c|c|c|}
\hline & & $\mathrm{F}$ & $\mathrm{R}_{\text {cal }}^{2}$ & SEC & SECV & $\mathrm{R}_{\text {val }}^{2}$ & SEP & Bias & RPD \\
\hline \multirow{3}{*}{$\mathrm{CV}$} & Raw spectra & 5 & 0.97 & 1.93 & 2.15 & 0.97 & 2.03 & -0.21 & 5.6 \\
\hline & First derivative spectra & 5 & 0.98 & 1.76 & 1.95 & 0.97 & 1.96 & -0.23 & 5.7 \\
\hline & Second derivative spectra & 5 & 0.98 & 1.68 & 1.87 & 0.97 & 1.93 & -0.15 & 5.8 \\
\hline
\end{tabular}

F, number of factors; R, correlation coefficient; SEC, standard error of calibration; SECV, standard error of cross validation; SEP, standard error of prediction; Bias, average of differences between reference value and FTIR predicted value; $R P D$, ratio of standard deviation of reference data in the validation set to SEP 


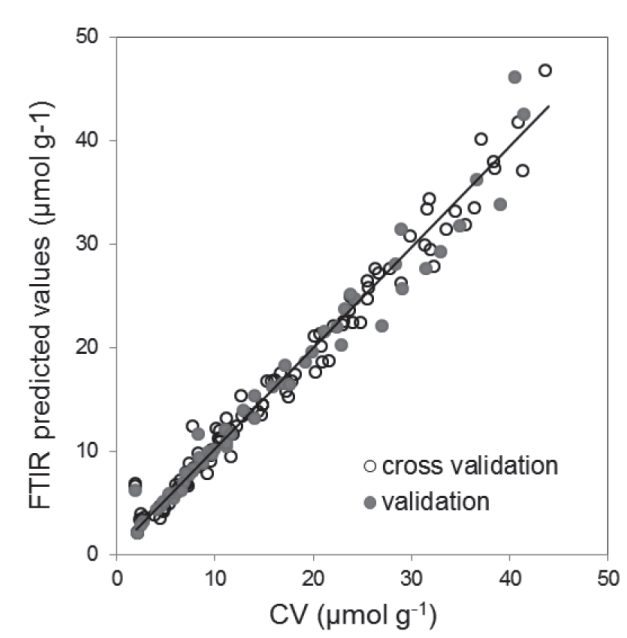

Fig. 3 Relationship between reference CVs and FTIRpredicted values.

the development of a robust calibration model. The first derivative of the raw spectra is simply a measure of the slope of the spectral curve at every point and the second derivative is a measure of the change in the slope of the curve. The slope of the curve is not affected by baseline offsets in the spectrum, and thus using the first and second derivatives are very effective methods for removing baseline offsets ${ }^{22,23)}$. This may be why relatively better results were obtained based on the first or second derivative spectra.

The best calibration model was obtained using the second derivative spectra of frying oils. For CV, the model showed low values of $\operatorname{SECV}\left(1.87 \mu \mathrm{mol} \mathrm{g}{ }^{-1}\right)$ and $\operatorname{SEP}(1.93$ $\left.\mu \mathrm{mol} \mathrm{g}^{-1}\right)$. Figure 3 shows the strong linearity between the cross-validation and prediction (validation) results, represented graphically by plotting the reference analysis values against the IR-predicted values based on the second derivative spectra. The prediction model also had high ratio performance deviation (RPD) values of more than 5.8. Generally, an RPD value above 3 indicates a useful model that allows good quantitative predictions ${ }^{28-30)}$. It can therefore be concluded that the IR spectra provided good estimations of CV in frying oils.

\subsection{Regression coefficients of PLS model}

Regression coefficients can be used to compare the contributions of individual wavenumbers to a PLS calibration model, since a regression coefficient spectrum shows characteristic peaks and troughs that can indicate the wavenumber range that is important for the calibration mode ${ }^{31-34)}$.

Figure 4 show the regression coefficients of the PLS calibration model based on the second derivative spectra for CV values. Some notable peaks at wavenumbers of 3026 , 3008, 1736, 1147 and $968 \mathrm{~cm}^{-1}$ were easily observed. The negative peak at a wavenumber of $1736 \mathrm{~cm}^{-1}$ could be assigned to the $\mathrm{C}=\mathrm{O}$ functional group, which might be

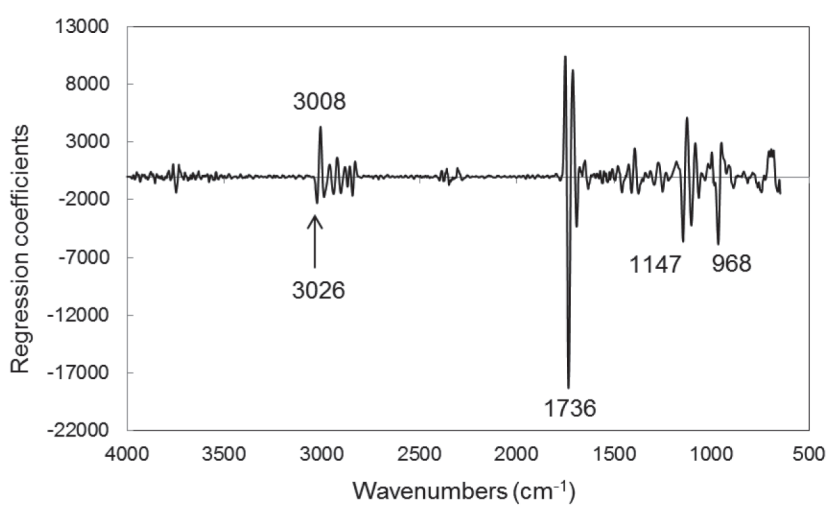

Fig. 4 Regression coefficients of the PLS calibration model for $\mathrm{CV}$, based on the second derivative spectra.

related to the absorption of the $\mathrm{C}=\mathrm{O}$ stretching characteristic frequency associated with aldehyde and ketone whose content increases with the degradation of frying oils ${ }^{17,20,35,36)}$. The negative peak at a wavenumber of $3026 \mathrm{~cm}^{-1}$ could be assigned to the $\mathrm{C}-\mathrm{H}$ trans functional group and the positive peak at a wavenumber of $3008 \mathrm{~cm}^{-1}$ could be assigned to the $\mathrm{C}-\mathrm{H}$ cis functional group, which might be related to the increase in trans fatty acids and the relative decrease in cis fatty acids when frying oils degrade ${ }^{17,20,35,36)}$. The negative peak at a wavenumber of $1147 \mathrm{~cm}^{-1}$ might be assigned to the C-O stretching characteristic frequency or $\mathrm{CH}_{2}$ bending frequency ${ }^{17,20,35,36)}$. The negative peak at a wavenumber of $968 \mathrm{~cm}^{-1}$ could be assigned to $\mathrm{HC}=\mathrm{CH}$ trans bending (outof-plane) functional group, which is related to the absorption of trans fatty acids, which increases with the degradation of frying oils ${ }^{17,20,35,36)}$.

These results show that PLS calibration models for CV of frying oils can be established based on the absorptions of deterioration products such as carboxylic acid, aldehyde, ketone groups and unsaturated fatty acids, which increase as frying oil deteriorate. The results of the present study have shown the utility of using the IR technique for rapidly determining the CV of frying oils. However, when the calibration got in this paper applies to other types of frying oil, a further research using the types of frying oil is necessary to confirm the adaptability.

\section{CONCLUSIONS}

FTIR spectroscopy can be successfully applied for determining the CV of frying oils. A good calibration model, based on the second derivative spectra, gave a low SEP value $\left(1.93 \mu \mathrm{mol} \mathrm{g}^{-1}\right)$ and a high $\mathrm{RPD}$ value (5.8) for predicting CV. FTIR spectroscopy has significant advantages over other chemical analysis techniques; it is fast and simple and requires no sample preparation, so is a very practical method for measuring the $\mathrm{CV}$ of frying oils. 


\section{References}

1) Blumenthal, M. M. A new look at the chemistry and physics of deep-fat frying. Food Technol. 45, 68-71 (1991).

2) White, P. J. Methods for measuring changes in deepfat frying oils. Food Technol. 45, 75-80 (1991).

3) Clark, W. L.; Serbia, G. W. Safety aspects of frying fats and oils. Food Technol. 45, 84-89 (1991).

4) Tyagi, V. K.; Vasishtha, A. K. Changes in the characteristics and composition of oils during deep-fat frying. J. Am. Oil Chem. Soc. 73, 499-506 (1996).

5) Frankel, E. N. Secondary products of lipid oxidation. Chem. Phys. Lipids 44, 73-85(1987).

6) Chiba, T.; Takazawa, M.; Fujimoto, K. A simple method for estimating carbonyl content in peroxide-containing oils. J. Am. Oil Chem. Soc. 66, 1588-1592(1989).

7) Esterbauer, H.; Schaur, R. D.; Zollner, H. Chemistry and biochemistry of 4-hydroxynonenal, malonaldehyde and related aldehydes. Free Radical Biol. Med. 11, 81-128 (1991).

8) Endo, Y.; Li, C. M.; Tagiri-Endo, M.; Fugimoto, K. A modified method for the estimation of total carbonyl compounds in heated and frying oils using 2-propanol as a solvent. J. Am. Oil Chem. Soc. 10, 1021-1024 (2001).

9) Henick, A. S.; Benca, M. F.; Mitchell, J. H. Estimating carbonyl compounds in rancid fats and foods. J. Am. Oil Chem. Soc. 31, 88-91 (1954).

10) Kumazawa, H.; Oyama, T. Estimation of total carbonyl content in oxidized oil by 2,4-dinitrophenylhydrazine. J. Am. Oil Chem. Soc. 14, 167-171 (1965).

11) Endo, Y.; Tominaga, M.; Tagiri-Endo, M.; Kumozaki, K.; Kouzui, H.; Shiramasa, H.; Miyakoshi, K. A modified method to estimate total carbonyl compounds in frying oils using 1-butanol as a solvent. J. Oleo Sci. 52, 353-358 (2003).

12) Li-Chan, E.; Ismail, A.; Sedman, J.; Voort, F.R. Vibrational spectroscopy of food and food products. In J. M. Chalmers \& P. R. Griffiths (Eds.). Handbook of Vibrational Spectroscopy 3629-3662 (2002).

13) Permanyer, A.; Douifi, L.; Lahcini, A.; Lamontagne, J.; Kister, J. FTIR and SUVF spectroscopy applied to reservoir compartmentalization: a comparative study with gas chromatography fingerprints results. Fuel 84, 159-168 (2005).

14) Nunes, C. A. Vibrational spectroscopy and chemometrics to assess authenticity, adulteration and intrinsic quality parameters of edible oils and fats. Food Res. Int. 60, 255-261 (2014).

15) Maggio, R. M.; Kaufman, T. S.; Carlo, D.; Cerretani, L.; Bendini, A.; Cichelli, A.; Compagnone, D. Monitoring of fatty acid composition in virgin olive oil by Fourier transformed infrared spectroscopy coupled with partial least squares. Food Chem. 114, 1549-1554
(2009).

16) Wang, L.; Lee, F.; Wang, X.; He, Y. Feasibility study of quantifying and discriminating soybean oil adulteration in camellia oils by attenuated total reflectance MIR and fiber optic diffuse reflectance NIR. Food Chem. 95, 529-536(2006).

17) Lerma-García, M. J.; Ramis-Ramos, G.; HerreroMartínez, J. M.; Simó-Alfonso, E. F. Authentication of extra virgin olive oils by Fourier-transform infrared spectroscopy. Food Chem. 118, 78-83 (2010).

18) Rohman, A.; Man, Y. B. C. Fourier transform infrared (FTIR) spectroscopy for analysis of extra virgin olive oil adulterated with palm oil. Food Res. Int. 43, 886$892(2010)$.

19) Hocevar, H.; Soares, V.; Oliveira, F.; Korn, M.; Teixeira, L. Application of Multivariate Analysis in Mid-Infrared Spectroscopy as a Tool for the Evaluation of Waste Frying Oil Blends. J. Am. Oil Chem. Soc. 89, 781-786 (2012).

20) Zhang, Q.; Liu, C.; Sun, Z.; Hu, X.; Shen, Q.; Wu, J. Authentication of edible vegetable oils adulterated with used frying oil by Fourier Transform Infrared Spectroscopy. Food Chem. 132, 1607-1613 (2012).

21) JOCS. Tentative 13-2003 Carbonyl Value(Butanol Method). Standard Methods for the Analysis of Oils, Fats and Derivatives. Tokyo, JOCS (2003).

22) Duckworth, J. Mathematical data preprocessing. NearInfrared Spectroscopy in Agriculture, ed. Roberts et al. 115-132(2004).

23) Chen, J. Y.; Matsunaga, R.; Ishikawa, K.; Zhang, H. Main inorganic component measurement of seawater using near-infrared spectroscopy. Applied Spec. 57, 1399-1406 (2003).

24) Madden H. M. Comments on the Savitsky-Golay convolution method for least-squares-fit smoothing and differentiation of digital data. Anal. Chem. 50, 1383-1386 (1978).

25) Vlachos, N.; Skopelitis, Y.; Psaroudaki, M.; Konstantinidou, V.; Chatzilazarou, A.; Tegou, E. Applications of Fourier transform-infrared spectroscopy to edible oils. Anal. Chem. Acta 573574, 459-465 (2006).

26) Al-Degs, Y.; Al-Ghouti, M.; Salem, N. Determination of Frying Quality of Vegetable Oils used for Preparing Falafel using Infrared Spectroscopy and Multivariate Calibration. Food Anal. Methods 4, 540-549(2011).

27) Yang, H.; Irudayaraj, J. Characterization of semisolid fats and edible oils by Fourier transform infrared photoacoustic spectroscopy, J. Am. Oil Chem. Soc. 77, 291-295 (2000).

28) Williams, P. "Near-infrared technology in the agricultural and food industries", "Implementation of near-infrared technology", $A A C C$, Chapter 8, 145-169 (2001). 
29) Chen, J. Y.; Zhang, H.; Miao, Y.; Matsunaga, R. NIR measurement of specific gravity of potato. Food Sci. Technol. Res. 11, 26-31 (2005).

30) Chen, J. Y.; Zhang, H.; Matsunaga, R. Visible and Near Infrared Spectroscopy for Rapid Analysis of the Sugar Composition of Raw Ume Juice. Food Sci. Technol. Res. 13, 291-295 (2007).

31) Martens, H.; Naes, T. Multivariate Calibration. John Wiley and Sons, Chichester, UK. (1989).

32) Chen, J. Y.; Miao, Y.; Zhang, H.; Matsunaga, R. Nondestructive determination of carbohydrate content in potatoes using near infrared spectroscopy. J. Near Infrared Spec. 12, 311-314(2004).

33) Chen, J. Y.; Zhang, H.; Matsunaga, R. Rapid determination of the main organic acid composition of raw Japanese apricot fruit juices using near-infrared spectroscopy. J. Agric. Food Chem. 54, 9652-9657 (2006).

34) Chen, J. Y.; Miao, Y.; Sato, S.; Zhang, H. Near Infrared Spectroscopy for determination of the Protein Composition of Rice Flour. Food Sci. Technol. Res. 14, 132-138 (2008).

35) Guillen, M. D.; Cabo, N. Characterization of edible oils and lard by Fourier transform infrared spectroscopy. Relationships between composition and frequency of concrete bands in the fingerprint region. J. Am. Oil Chem. 74, 1281-1286(1997).

36) Lerma-Garcia, M. J.; Simo-Alfonso, E. F.; Bendini, A.; Cerretani, L. Rapid evaluation of oxidized fatty acid concentration in virgin olive oil using Fouriertransform infrared spectroscopy and multiple linear regression. Food Chem., 124, 679-684(2011). 